\title{
Bifurcation and Voltage C ollapse in the Electrical Power Systems
}

\author{
Mr. Ahmed N. B. Alsammak, M.Sc. \\ Electrical Engineering Department \\ University of Mosul \\ Mosul - Iraq
}

\begin{abstract}
:
Voltage stability is indeed a dynamic problem. Dynamic analysis is important for a better understanding of voltage instability process. In this work an analysis of voltage stability from bifurcation and voltage collapse point of view based on a center manifold voltage collapse model. A static and dynamic load models were used to explain voltage collapse. The basic equations of a simple power system and load used to demonstrate voltage collapse dynamics and bifurcation theory. These equations are also developed in a manner, which is suitable for the Matlab-Simulink application. As a result detection of voltage collapse before it reach the critical collapse point was obtained as original point.
\end{abstract}

Keywords: Power System Stability, Voltage Stability, Voltage Collapse, Bifurcation, Reactive Power Compensation and Matlab-Simulink.

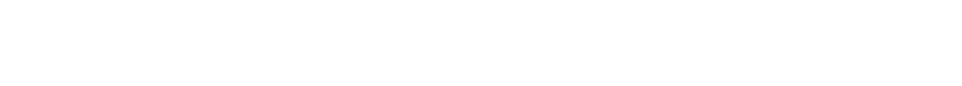

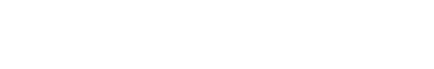

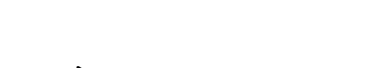

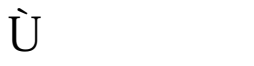

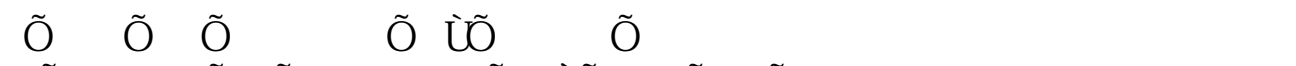
:ŭ ŤG:E

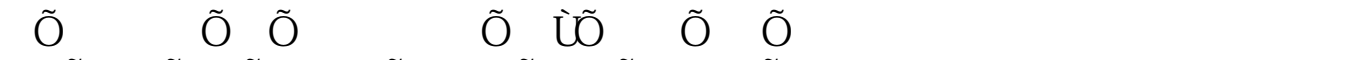

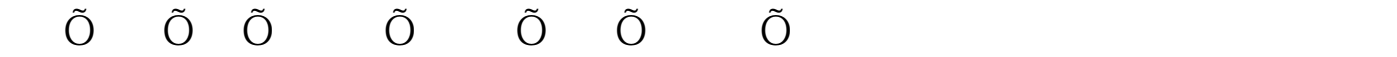

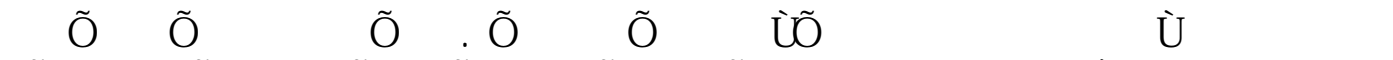

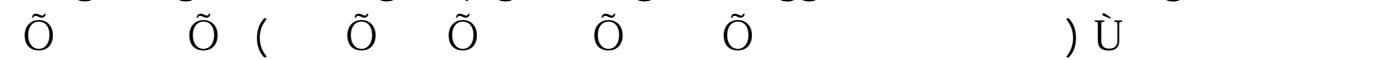
औA

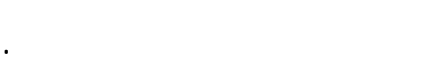




\section{List of symbols:}

$\mathrm{V}=$ Amplitude terminal load voltage (p.u.).

$\delta=$ Internal terminal load voltage angle in degree.

$\mathrm{Em}=$ Amplitude of generator internal voltage (p.u.).

$\delta \mathrm{m}=$ Internal generator voltage angle in degree.

Eo $=$ infinity bus or slack bus voltage (p.u.).

$\mathrm{C}=$ compensated load capacitor in p.u.

Yo $=$ Amplitude of equivalent impedance for the transformer and transmission line in p.u.

$\mathrm{Ym}=$ Amplitude of equivalent impedance for the generator, transformer and transmission line in p.u.

$\mathrm{M}=$ Generator moment of inertia p.u.

$\mathrm{dm}=$ damping coefficient

$\mathrm{Pm}=$ Mechanical power.

$\mathrm{P} \& \mathrm{Q}=$ Real and reactive power load demand respectively.

$\mathrm{K}_{\mathrm{pw}}, \mathrm{K}_{\mathrm{pv}}, \mathrm{K}_{\mathrm{qw}}, \mathrm{K}_{\mathrm{qv}}$ and $\mathrm{K}_{\mathrm{qv} 2}=$ Constant parameters for the real and reactive load power.

$\omega=$ Speed and equal to $\dot{\delta}$.

\section{Introduction:}

The continuing interconnections of bulk power systems, brought about by economic and environmental pressures, have led to an increasingly complex system that must operate ever closer to limits of stability. This operating environment has contributed to the growing importance of the problems associated with the dynamic stability assessment of power systems. To a large extent, this is also due to the fact that most of the major power system breakdowns are caused by problems relating to the system dynamic responses. It is believed that new types of instability emerge as the system approaches the limits of stability.

One type of system instability, which occurs when the system is heavily loaded, is voltage collapse. This event is characterized by a slow variation in the system operating point, due to increase in loads, in such a way that voltage magnitudes gradually decrease until a sharp, accelerated change occurs.

Voltage collapse in electric power systems has recently received significant attention in the literature (see, e.g., [1] for a synopsis), this has been attributed to increases in demand which result in operation of an electric power system near its stability limits. A number of physical mechanisms have been identified as possibly leading to voltage collapse. From a mathematical perspective, voltage collapse has been viewed as arising from a bifurcation of the power system governing equations as a parameter is varied through some critical value. In several papers [9-15], voltage collapse is viewed as an instability which coincides with the disappearance of the steady state operating point as a 
system parameter, such as a reactive power demand is quasistatically varied. In the language of bifurcation theory, these papers link voltage collapse to a fold or saddle node bifurcation of the nominal equilibrium point.

Dobson and Chiang [2] presented a mechanism for voltage collapse, which postulates that this phenomenon occurs at a saddle node bifurcation of equilibrium points. They employed the Center Manifold Theorem to understand the ensuing dynamics, In the same paper., they introduced a simplex example power system containing a generator, an infinite bus and a nonlinear load (as shown in Fig.(1)). The saddle node bifurcation mechanism for voltage collapse postulated in Ref.[2] was investigated for this example in [3] and in [4].

All essential distinction exists between the mathematical formulation of voltage collapse problems and transient stability problems. In studying transient stability [5,6], one often interested in whether or not a given power system can maintain synchronism (stability) after being subjected to a physical disturbance of moderate or large proportions. The faulted power system in such a case has been perturbed in a severe way from steady-state, and one studies the possibility of the post-fault system returning to steady-state (regaining synchronism). In the voltage collapse scenario, however, the disturbance may be viewed as a slow change in a system parameter, such as a power demand. Thus, the disturbance does not necessarily perturb the system away from steady-state. The steady-state varies continuously with the changing system parameter until it disappears at a saddle node bifurcation point. It is therefore not surprising that saddle node bifurcation is being studied as a possible route to voltage collapse [7].

In this paper a suitable model is set up to analyze the power system in [2]. This model is then used with the some cases such as change in load and in the reactive load power as well as using constant and dynamic load, as induction motor.

The basic equations of the power system and load are also developed in a manner, which is suitable for the Matlab-Simulink application [8] and not depended on ready programs (compact program package) such as Auto [16]. The computer results show that voltage collapse may be studied before bifurcation with a static model and after bifurcation with a dynamic model so the goal of this work is to show the richness of the qualitative behaviors, which may occur near voltage collapse, and to illustrate their effect on system trajectories.

\section{Saddle-Node Bifurcations \&Voltage Collapse}

A saddle-node bifurcation is the disappearance of a system equilibrium as parameters change slowly. The saddle-node bifurcation of mot interest to power system engineers occurs when a stable equilibrium at which the power system operates disappears [1]. The consequence of this loss of the operating equilibrium is that the system state changes dynamically. In particular, the dynamics can be such that the system voltages fall in a voltage collapse. Since a saddle-node bifurcation can cause a voltage collapse there for it is useful to study saddle-node bifurcations of power system models in order to avoid these collapses, such as using PID controller to control saddle-node bifurcations [17]. 


\section{Reactive Power and Voltage Collapse:}

Voltage collapse typically occurs in power systems which are heavily loaded, faulted and/or have reactive power shortages. Voltage collapse is system instability that it involves many power system components and their variables at once. Indeed, voltage collapse often involves an entire power system, although it usually has a relatively larger involvement in one particular area of the power system [1].

Although many other variables are typically involved, some physical insight into the nature of voltage collapse may be gained by examining the production, transmission and consumption of reactive power. Voltage collapse is associated with the reactive power demands of loads not being met because of limitations in the production and transmission of reactive power. Limitations are the productions of reactive power include generator and SVC reactive power limits and the reduced reactive power produced by capacitors at low voltages. The primary limitations on the transmission of reactive power are the high reactive power loss on heavily loaded lines and line outages. Reactive power demands of loads increases with the increasing of load, motor stalling, or changes in load composition such as an increased proportion of compressor load.

\section{The Model}

This section summarizes an example from [4] to illustrate how voltage collapse model applies to the power system model shown in Fig.(1). One generator is a slack bus and the other generator has constant voltage magnitude $\mathrm{E}$, and angle dynamics given by the swing equation:

$$
M \cdot \ddot{\delta} m=-d m \cdot \omega+P m+E m \cdot V \cdot Y m \cdot \sin (\delta-\delta m-\theta m)+E m^{2} \cdot Y m \cdot \sin (\theta m)
$$

where $M, d m$, and $P m$, are the generator moment of inertia, damping coefficient and mechanical power respectively.

The load model includes a dynamic induction motor based on a model of Walve [13] with a constant PQ load in parallel. The induction motor model specifies the real and reactive power demands $\mathrm{P}$ and $\mathrm{Q}$ of the motor in terms of load voltage $\mathrm{V}$ and frequency $\dot{\delta}$. The combined model for the motor and the PQ load [2] is:

$$
\begin{aligned}
& P=P o+P 1+K_{p w} \cdot \dot{\delta}+K_{p V}(V+T \cdot \dot{V}) \\
& Q=Q o+Q 1+K_{q w} \cdot \dot{\delta}+K_{q V} \cdot V+K_{q V 2} \cdot V^{2}
\end{aligned}
$$

where $P o, Q o$ are the constant real and reactive powers of the motor and $P 1, Q 1$ represent the $P Q$ load.

From eq.(4.3):

$$
\dot{\delta}=\frac{-K q v \cdot V-K q v 2 \cdot V^{2}+Q-Q o-Q 1}{K q w}
$$

Substituted eq. (4.4) in eq.(4.2) we get:

$$
\dot{V}=\frac{K p w K q \imath \cdot V^{2}+(K p w K q v-K q w K p v \cdot V+K p w(Q o+Q 1-Q)-K q w(P o+P 1-P)}{T \cdot K q w K p v} \text {. }
$$


thus,

$$
\dot{\delta} m=\omega m
$$

From eq.(4.1)\&(4.6) we get:

$$
\dot{\omega} m=\frac{-d m \cdot \omega m+P m+E m \cdot V \cdot Y m \cdot \sin (\delta-\delta m-\theta m)+E m^{2} \cdot Y m \cdot \sin (\theta m)}{M}
$$

In eq.(4.3) $Q 1$ is chosen as the system parameter so that increasing $Q 1$ corresponds to increasing the load reactive power demand. The load also includes a capacitor $\mathrm{C}$ as part of its constant impedance representation in order to maintain the voltage magnitude at a normal and reasonable value. It is convenient to derive the Thevenin equivalent circuit with the capacitor. The adjusted values are:

$$
\begin{aligned}
& E o^{\prime}=\frac{E o}{\sqrt{1+\frac{C^{2}}{Y o^{2}}-\frac{2 \cdot C}{Y o} \cdot \cos (\theta o)}} \\
& Y o^{\prime}=Y o \cdot \sqrt{1+\frac{C^{2}}{Y o^{2}}-\frac{2 \cdot C}{Y o} \cdot \cos (\theta o)} \\
& \theta o^{\prime}=\theta o+\tan ^{-1}\left(\frac{\frac{C}{Y o} \cdot \sin (\theta o)}{1-\frac{C}{Y o} \cdot \cos (\theta o)}\right)
\end{aligned}
$$

The real and reactive powers supplied to the load by the network are:

$$
\begin{aligned}
P(\delta, V) & =-E \delta^{\prime} \cdot Y \delta^{\prime} \cdot V \cdot \sin \left(\delta+\theta o^{\prime}\right)-E m \cdot V \cdot Y m \cdot \sin (\delta-\delta m+\theta m) \\
& +V^{2} \cdot\left(Y \delta^{\prime} \cdot \sin \left(\theta o^{\prime}\right)+Y m \cdot \sin (\theta m)\right) \\
Q(\delta, V) & =E \delta^{\prime} \cdot Y \delta^{\prime} \cdot V \cdot \cos \left(\delta+\theta o^{\prime}\right)+E m \cdot V \cdot Y m \cdot \cos (\delta-\delta m+\theta m) \\
& -V^{2} \cdot\left(Y \delta^{\prime} \cdot \cos \left(\theta o^{\prime}\right)+Y m \cdot \cos (\theta m)\right)
\end{aligned}
$$

In order to compute bifurcation value Q1 and the associated bifurcation equilibrium point, the following approximate formulas [15] are used as shown in appendix (A) equation (A3). The bifurcation value is:

$Q o+Q 1-\left(-K q v+E o^{\prime} \cdot Y o^{\prime}+E m \cdot Y m\right) \cdot V+\left(K q v 2+Y o^{\prime}+Y m\right) \cdot V^{2}=0$.

and the voltage magnitude at the bifurcation equilibrium point is:

$$
Q_{1}^{*}=\frac{\left(-K q v+E o^{\prime} \cdot Y o^{\prime}+E m \cdot Y m\right)^{2}}{4 \cdot\left(K q v 2+Y o^{\prime}+Y m\right)}-Q o
$$

Formulas (4.13) and (4.14) are derived from the approximate static model given in Ref.[15]:

$$
V^{*}=\frac{\left(-K q v+E o^{\prime} \cdot Y o^{\prime}+E m \cdot Y m\right)}{4 \cdot\left(K q v 2+Y o^{\prime}+Y m\right)}
$$

The last three equations show the relationship between the bifurcation point and certain load, transmission network and generator parameters. 


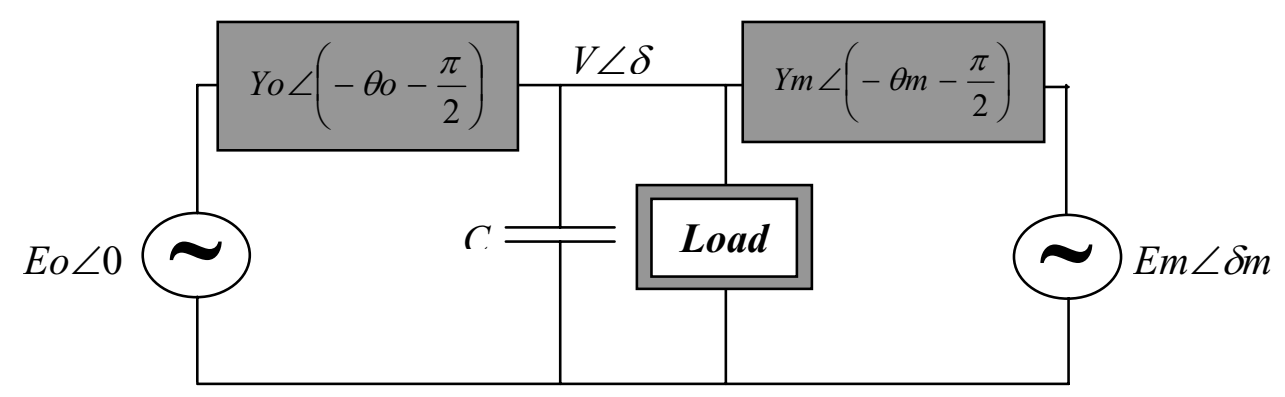

Fig.(1) Power System Model.

\section{Bifurcations}

Consider the modified power system model described by Ref.[2] which is given by (4.1), (4.2)\&(4.3) in the general form:

$\dot{x}=F(x, \lambda)$

where $x$ is the state vector and $\lambda$ is a time-varying parameter vector. Specifically, in the power system model described in section $(4), \mathrm{x}=(\delta, \omega, \mathrm{V})$ and $\lambda$ denotes the parameter vector that includes real and reactive power demands at each load bus. The parameters in (5.1) are subject to variation and, as a result, changes may occur in the qualitative structure of the solutions of the static equation associated with (5.1), i.e., solutions of $\mathrm{F}(x, \lambda)=0$ for certain values of $\lambda$. For example, a change in the number of solutions for $x$ may occur as the parameters vary. As a result, the dynamic behavior of (5.1) may be altered.

Bifurcation theory [1] is concerned with branching of the static solutions of (5.1) and, in particular, it is interested in how solutions $x(\lambda)$ branch as $\lambda$ varies. These changes, when they occur, are called Bifurcations and the parameter values at which a bifurcation happens are called bifurcation values.

It is important in our following analysis of voltage collapse to distinguish two different periods: the period before bifurcation and the period after bifurcation. Power systems are normally operated near a stable equilibrium point. As system parameters change slowly, the stable equilibrium point changes position but remains a stable equilibrium point. This situation may be modeled with the static model $\mathrm{F}(x, \lambda)=0$ by regarding $\mathrm{F}(x, \lambda)=0$ as specifying the position of the stable equilibrium point $x$ as a function of $\lambda$. (Here it would be more precise to call $\mathrm{F}(x, \lambda)=0$ a quasistatic model since $\lambda$ varies and causes corresponding variations in $(x)$. This model may also be called parametric load flow model. Exceptionally, variation in $\lambda$ will cause the stable equilibrium point to bifurcate. The stable equilibrium point of (5.1) may then disappear or become unstable depending on the way in which the parameter is varied and the specific structure of the system.

After the bifurcation, the system state will evolve according to the dynamics of (5.1). (Some types of bifurcation result in the persistence of the stable equilibrium point even after the bifurcation and the static model apply just as before the bifurcation. However, we do not expect this sort of bifurcation to be typical in power systems.) To summarize, analysis of a typical bifurcation of a 
stable equilibrium point in a power system with slowly moving parameters has two parts:

(1) Before the bifurcation when the (quasi) static model applies.

(2) After the bifurcation when the dynamical model (5.1) applies.

The current research on voltage collapse uses the static model and only considers the system before the bifurcation.

\section{Simulation Procedure}

In this work, the voltage stability procedure used to perform the simulation by the proposed model would be presented by a simple block diagram as shown in Fig.(2). The simulation have been made with the use of the step-by-step solution with using ode $15 \mathrm{~s}$, ordinary differential equations which used to solve stiff problem with good accuracy. The used program is Matlab 6.0 [8] to which fast and accuracy results could be obtained. The differential equations from 4.1 to 4.7 are arrangement in such away by using the following figure to the results.

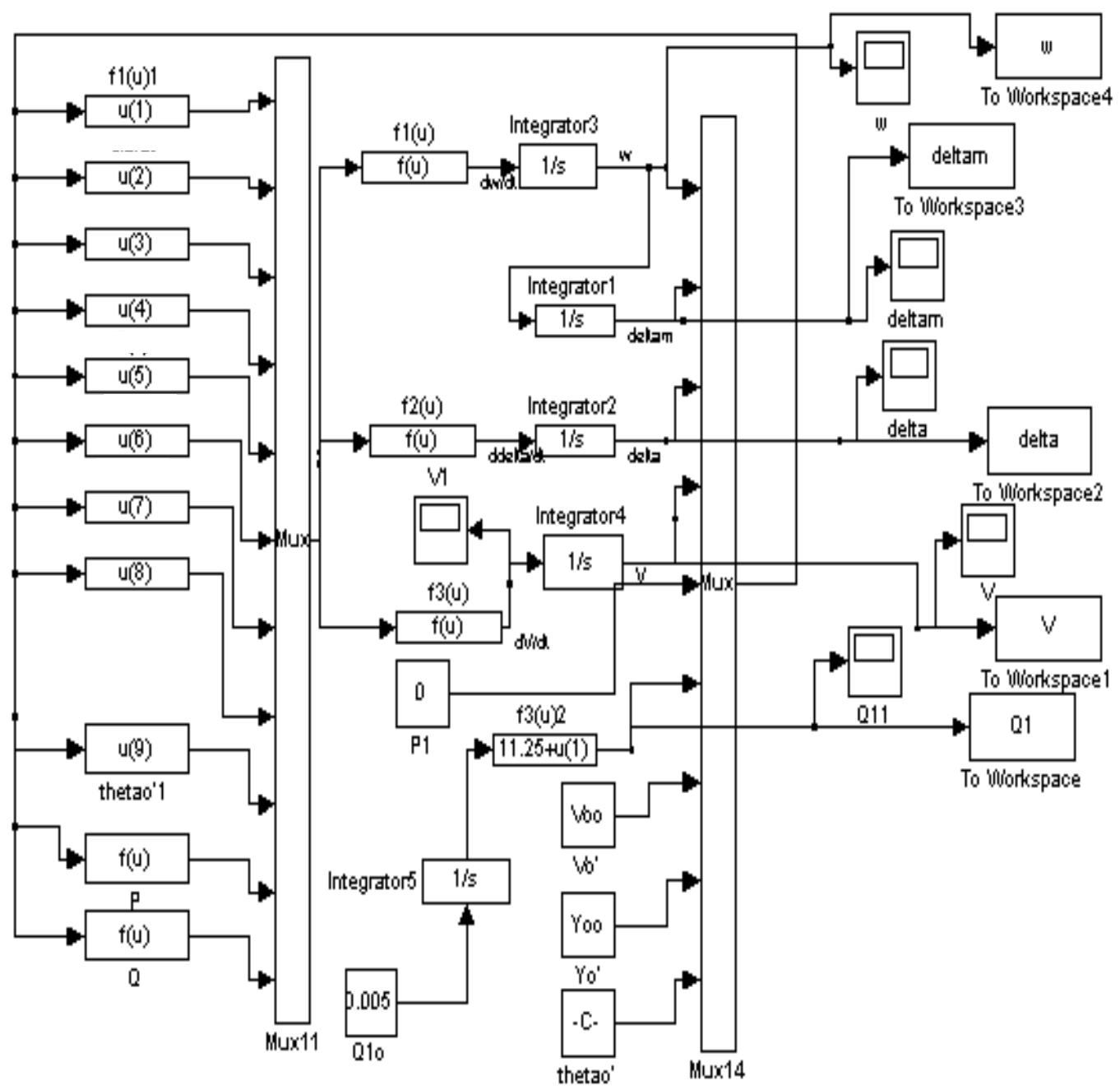

Fig.(2) Simulink model for the sample system. 


\section{Results and Discussion}

A model of the sample system shown in Fig.(1) and foregoing equations are used to illustrate the process of voltage collapse. For the bifurcations analysis, Fig.(3) shows the bifurcation diagram, which is appears the relates between voltage magnitude $\mathrm{V}$ and reactive power demand $Q 1$. This figure investigates a generic mechanism leading to disappearance of stable equilibrium points and the consequent system dynamics for one-parameter dynamical systems. To simplify the discussion, note first that in Fig.(3) which shows the relation between six bifurcation's depicted. For $Q 1<10.95$, a stable equilibrium point exists. (Upper left in Fig.(3)). As $Q 1$ is increased, an unstable ("subcritical") Hopf bifurcation is encountered at point $Q 1=10.95$. As $Q 1$ is increased further the stationary point regains stability at $Q 1=Q 1^{*}=11.42$ through a stable ("supercritical") Hopf bifurcation.

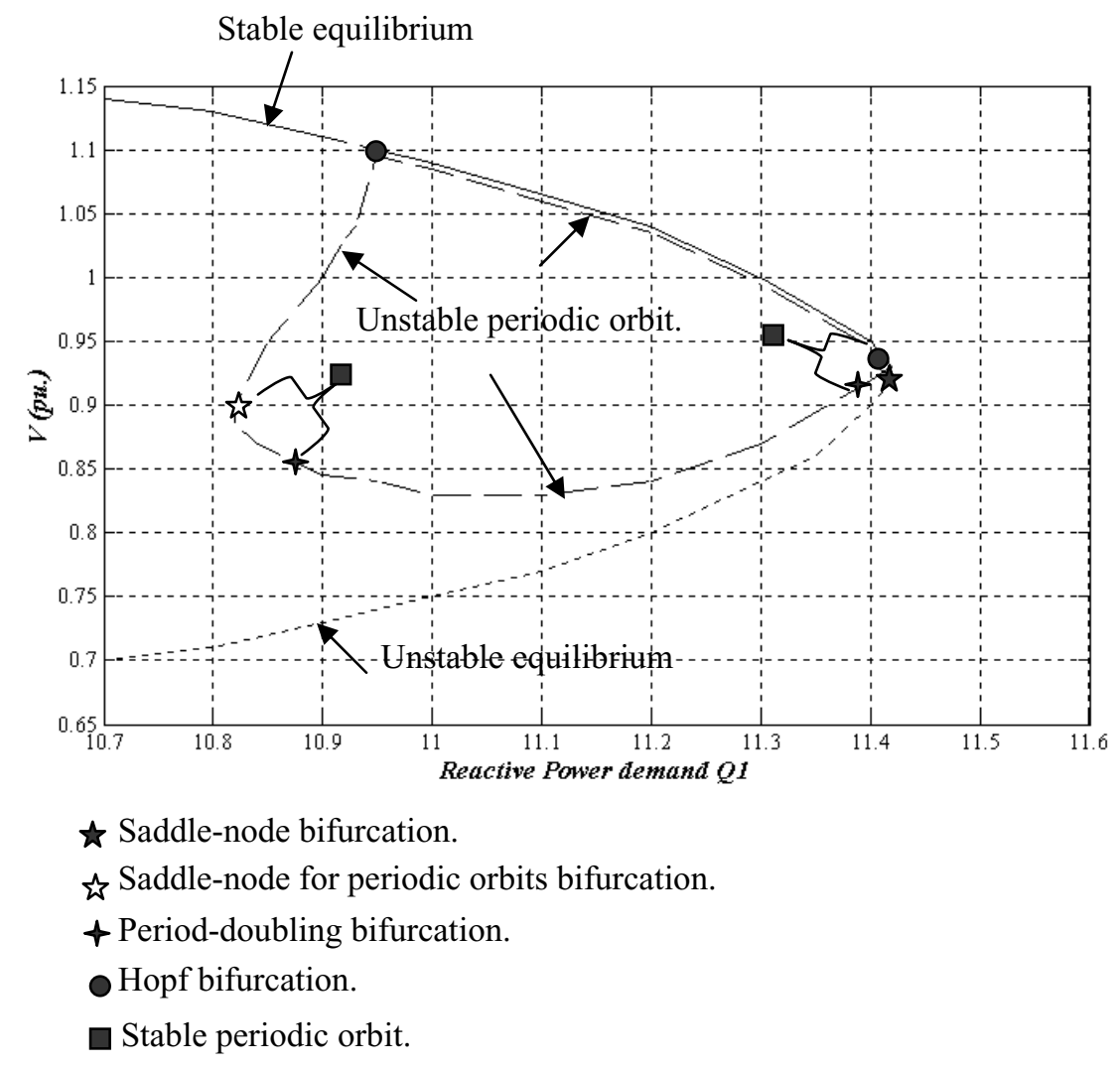

Fig.(3) Bifurcation diagram.

Fig.(4) shows an example of a typical voltage collapse, for the fourth order models, phenomenon after a saddle node bifurcation. The initial conditions used to generate the simulations are: $\delta m=0.35, \omega=0.001 \delta=0.14$, and $V=0.9$. Note the oscillatory nature of solution due to varying in $Q 1$, where $Q 1=11.25+0.005 t$ The previous example, Fig.(4), demonstrated the center manifold model for the 
dynamics of voltage collapse after a saddle node bifurcation. Now we simulate the behavior of the example both before and after the bifurcation to illustrate the entire process (we use the same set parameters as that in the above except $\mathrm{Ym}$ and capacitance $C$ changes with bifurcation value $Q 1^{*}, Q 1^{*}=Q 1$ at saddle node). The system can supply sufficient reactive power to the load while $Q 1<Q 1^{*}$. After the bifurcation, the behavior is similar to that predicted by the dynamical center manifold model. Fig.(5) shown the relationship between the transmission line parameter and the bifurcation value $\left(Q 1^{*}\right)$. This figure indicates that a larger transmission capacity ensures a larger bifurcation value.

One good and efficient way to increase the capacity of the power system is to using reactive power compensation such as using capacitors at load bus as shown in Fig.(1). The relationship between the bifurcation value of the system, which described in equations (4.1-4.15) and the amount of capacity installed at load bus is shown in Fig.(6), which shows also the effects on load voltage (V).

The previous figure, Fig.(3), shows what happens as the reactive power demand $Q 1$ increases. For $Q 1<10.95$ only a point stable (ps) and an point unstable (pu) equilibrium exit. Voltage collapse happens when a perturbation kicks the system out of the basin of attraction. Fig.(7) shows the relationship between $\delta m$, $\omega$, and $V$ where at the moment of bifurcation $\mathrm{W}^{\mathrm{u}}(\mathrm{pu})$, unstable manifold, no longer goes to ps but accumulates on $\Gamma \mathrm{u}$ instead. Therefore $\mathrm{W}^{\mathrm{u}}(\mathrm{pu})$ is contained in $\mathrm{W}^{\mathrm{s}}(\Gamma \mathrm{u})$, stable manifold, the basin of attraction of ps can no longer have the trumpet shape. For larger $Q 1$-values $\mathrm{W}^{\mathrm{u}}(\mathrm{pu})$ lies outside $\mathrm{W}^{\mathrm{s}}(\Gamma \mathrm{u})$ and is no longer part of the basin of attraction of ps. Fig.(8) shows the relationship between $\delta m$ and $\omega$ when the system goes toward voltage collapse.

Fig.(9) shows the stable manifold periodic orbit with long simulation time equal to $180 \mathrm{sec}$. In this case small disturbances cannot push the system out of the basin of attraction, i.e. the system always operates near ps.

\section{Conclusions}

Several voltage collapses are of slowly decreasing voltage nature followed by an accelerating collapse in voltage. In this paper we clarify the static and dynamic models used to explain this type of voltage collapse where the static model is used before a saddle-node bifurcation and the dynamic model is employed after the bifurcation.

Bifurcations have been studied for a power system dynamic model, which has previously been used to illustrate voltage collapse. It was found that for this model, the nominal operating point undergoes dynamic bifurcations prior to the static bifurcation to which voltage collapse has been attributed. These dynamic bifurcations result in a reduced stability margin in parameter space. Moreover, a short oscillatory voltage transient typically occurs prior to voltage collapse for this model. In addition, it was found that the model admits large amplitude bifurcations including cyclic fold and period doubling bifurcations; the latter leading to period doubling cascades and the resulting chaotic behavior. The relative importance of these effects in general power systems under stressed conditions is a topic for further research. 
Most of the previous analysis of voltage collapse has considered only the period before bifurcation. In this paper we show by an example that voltage collapse can be prospective before it happens and can be treated by reactive power control $(Q)$ or by load shedding $(P)$.

\section{Appendix (A): (Compute bifurcation value Q1)}

A saddle node bifurcation was found by solving equations $4.4,4.5$ and 4.7 with left hand side equal to zero as shown in Fig.(3). So from equation 4.4 can be obtain the following equation:

$-K q v \cdot V-K q v 2 \cdot V^{2}+Q-Q o-Q 1=0$

All angles at this point (saddle node) near to zero so equation 4.11 modified to $Q=E \delta \cdot Y \delta \cdot V+E m \cdot Y m \cdot V-V^{2} \cdot(Y d+Y m)$

Substituted equation (A2) in equation (A1) $Q o+Q 1-\left(-K q v+E o^{\prime} \cdot Y o^{\prime}+V m \cdot Y m\right) \cdot V+\left(K q v 2+Y o^{\prime}+Y m\right) \cdot V^{2}=0$

The voltage magnitude at the bifurcation equilibrium point are shown in the following equations, which driven from the approximate static model given in Ref.[15] and the above formulas, :

$$
\begin{aligned}
Q_{1}^{*} & =\frac{\left(-K q v+E o^{\prime} \cdot Y o^{\prime}+E m \cdot Y m\right)^{2}}{4 \cdot\left(K q v 2+Y o^{\prime}+Y m\right)}-Q o \\
V^{*} & =\frac{\left(-K q v+E o^{\prime} \cdot Y o^{\prime}+E m \cdot Y m\right)}{4 \cdot\left(K q v 2+Y o^{\prime}+Y m\right)}
\end{aligned}
$$

\section{Appendix (B):}

The sample power system parameter values used in the simulation are [2]: $K p w=0.4, K p v=0.3, K q \psi=-0.03, K q v=-2.8, K q \nu=2.1, T=8.5, P o=0.6, Q o=1.3$ $P 1=0.0, Y o=20.0, \theta o=-5.0, E o=1.0, C=12.0, Y o^{\prime}=8.0, \theta o^{\prime}=-12.0, E o^{\prime}=2.5, Y m=5.0$ $\theta m=-5.0, E m=1.0, P m=1.0, M=0.3, D=0.05$. All parameter values are in per unit except for angles, which are in degrees.

\section{References:}

[1] IEEE/PES Power System Stability Subcommittee Special Publication, "Voltage Stability Assessment, Procedures and Guides", Final Version, December 2000.

[2] I. Dobson and H.-D. Chiang, "Towards a theory of voltage collapse in electric power systems", Systems and Control Letters, Vol. 13, 1989, pp. 253-262.

[3] E. H. Abed, et al., "Dynamic bifurcations in a power system model exhibiting voltage collapse," Technical Research Report, University of Maryland, pp. 1-15, TR92-26, 25-Feb, 1992. 
[4] H. O. Wang, et al., "Bifurcations, Chaos and Crises in power system Voltage Collapse," Technical Research Report, University of Maryland, pp. 1-23, TR92-72, 25-Feb, 1992.

[5] Peter W. Sauer and M. A. Pai, " Power System Dynamics and Stability", Prentice Hall Inc. 1998.

[6] B. M. Weedy, Electric Power Systems. Great Britain: Wiley, 1972.

[7] E. H. Abed, et al., "On bifurcations in power system models and voltage collapse," in Proc. 29th Conf. Decision and Control, pp. 30143015, 1990.

[8] Matlab Works Inc. " Simulink (Dynamic System Simulation for MATLAB)", version 6.0, 2000.

[9] M. M. Begovic and A. G. Phadke, "Analysis of voltage collapse by simulation," Znt. Symp. Circuits and Systems, May 1989.

[10] K. T. Vu and C. C. Liu, "Dynamic mechanisms of voltage collapse", Systems and Control Letters, vol. 15, pp. 329-338, 1990.

[11] T. Van Cutsem, "A method to compute reactive power margins with respect to voltage collapse," LYEE Trans. Power Systems, vol. voltage collapse," LYEE Trans. Power Systems, vol. 6, pp. 145-156, Feb. 1991.

[12] H.-D. Chiang, C.-W. Liu, P.P- Varaiya, F.F. Wu and M.G. Lauby, "Chaos in a simple power system", Paper No.92 WM 151-1 PWRS, IEEE Winter Power Meeting, 1992.

[13] K. Walve, " Modeling of Power System Components at Severe Disturbance", CIGRE paper 38-18, International Conference on Large High Voltage Electrical Systems, 1986.

[14] K.T. Vu and C.C. Liu "Dynamic mechanisms of voltage collapse," Systems and Control letters, Vol. 15, 1990, pp. 329-338.

[15] I. Dobson and H.-D. Chiang, "A model of voltage collapse in electric power systems", IEEE Proceedings of $27^{\text {th }}$ Conference on Control and Decision, Dec.1988, Austin, Tx, pp. 2104-2109.

[16] EJ. Doedel, "AUTO: A program for the automatic bifurcation analysis of autonomous systems”, Cong. Num., Vol.30, pp. 265-284, 1981.

[17] D. Rosas, C Amaro and Alvarez, "Control of A Saddle Node Bifurcation in Power System using A PID Control", Journal of Applied Research and Technology, Vol.1, No.1, April 2003, pp.94-102. 


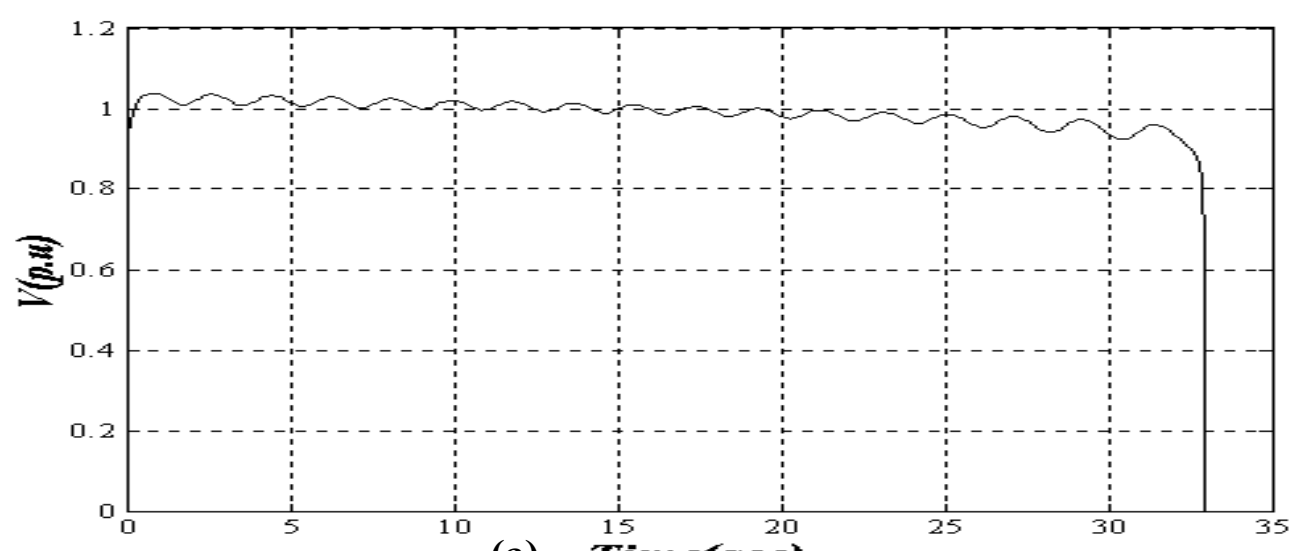

(a)

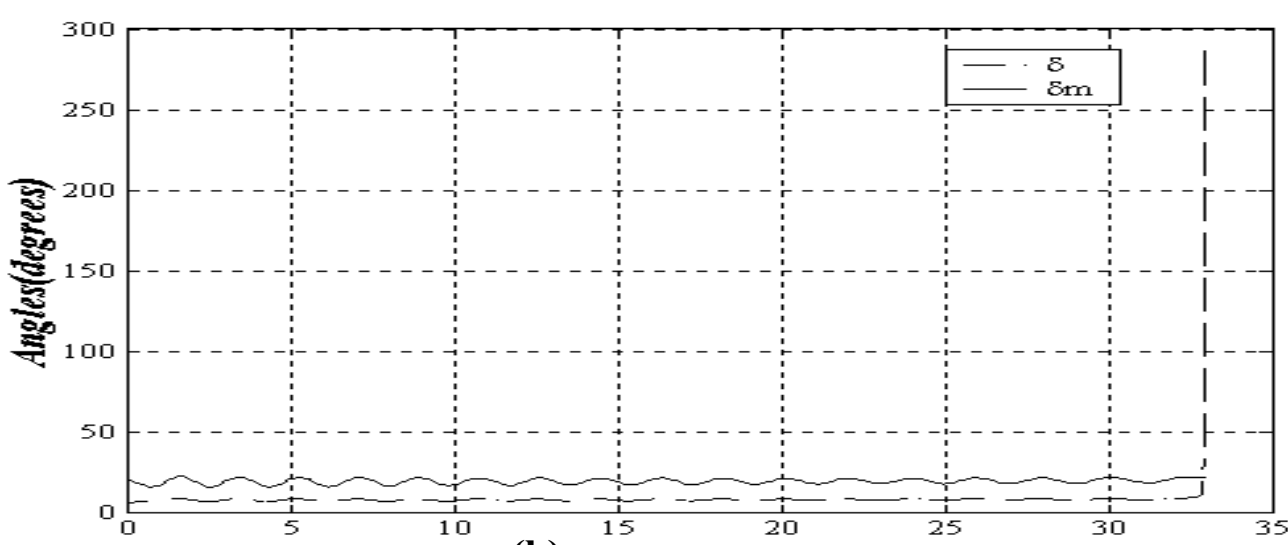

(b)

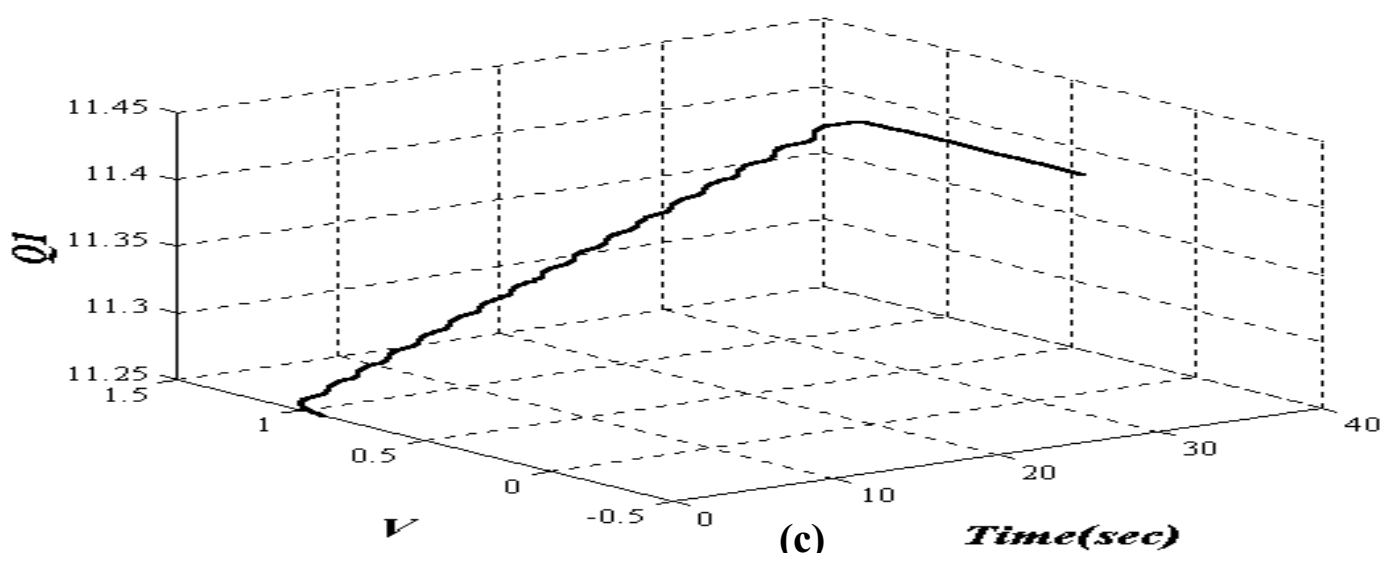

Fig.(4) Voltage magnitude, angles ( $\delta \mathrm{m}, \delta$ ), and varying Q1 with the time at load bus when bifurcation occurs. 


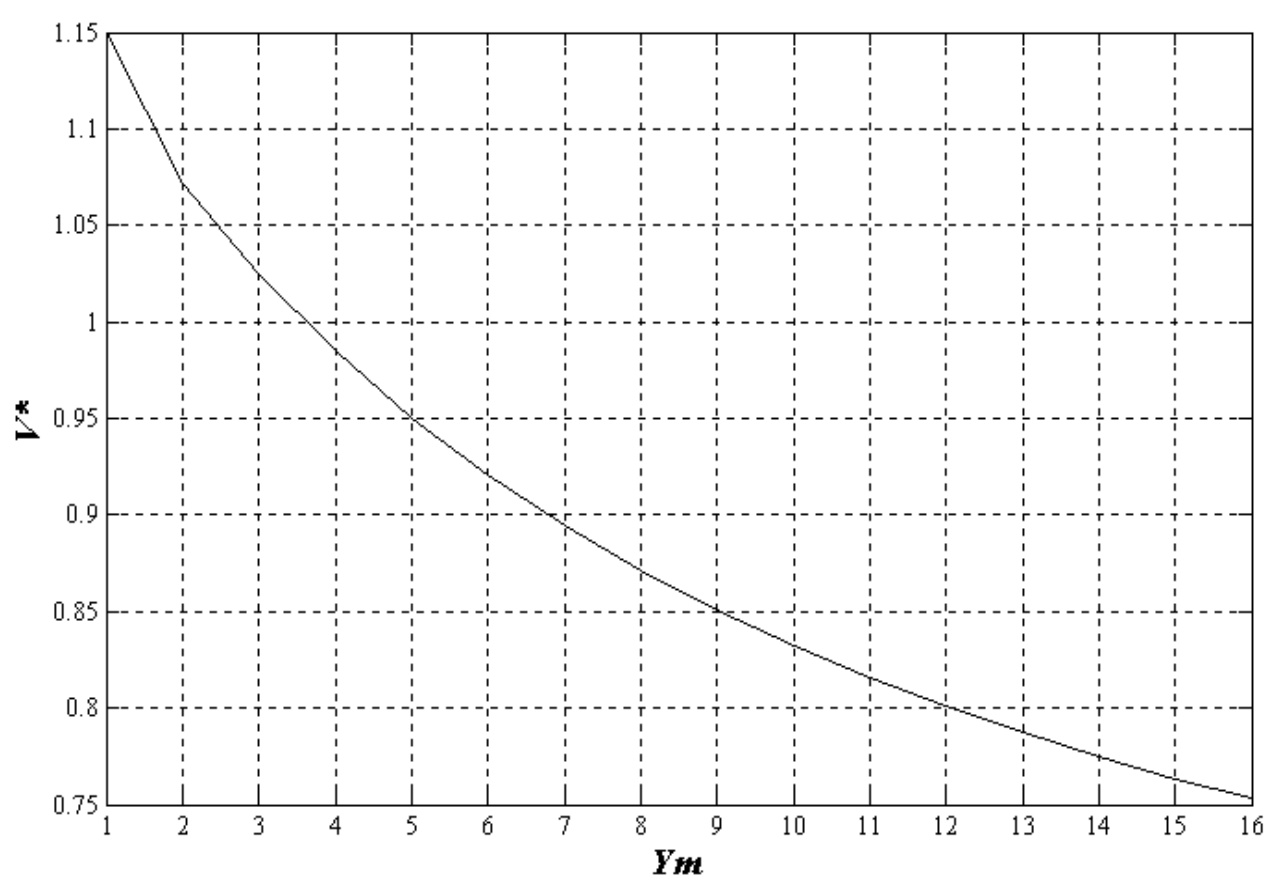

(a)

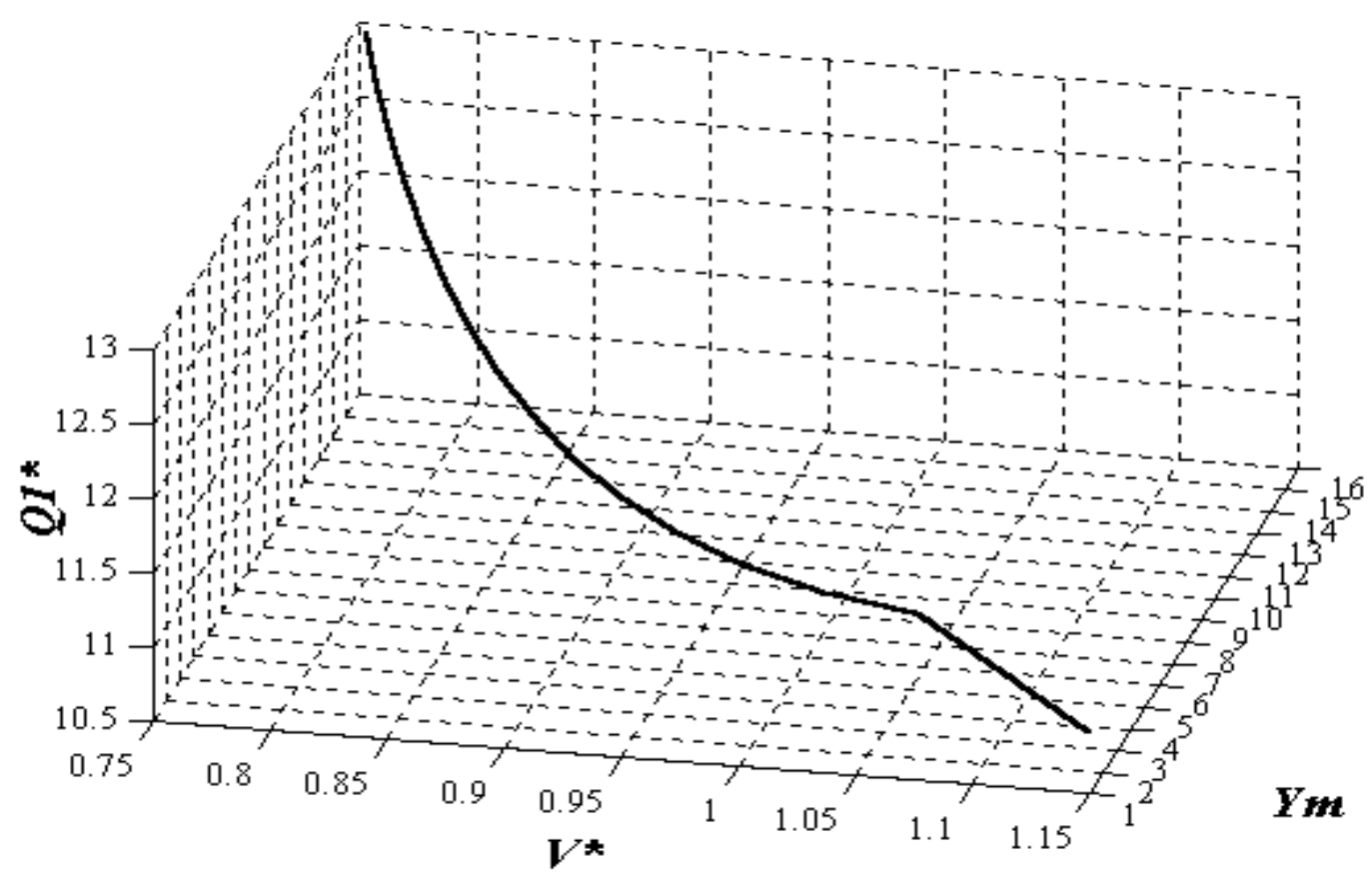

(b)

Fig.(5) Relationship between the transmission line parameter and:

Ine) Voltage magnitude at bifurcation point.

wo) The bifurcation value $\left(Q 1^{*}\right)$ and Voltage magnitude at bifurcation point. 


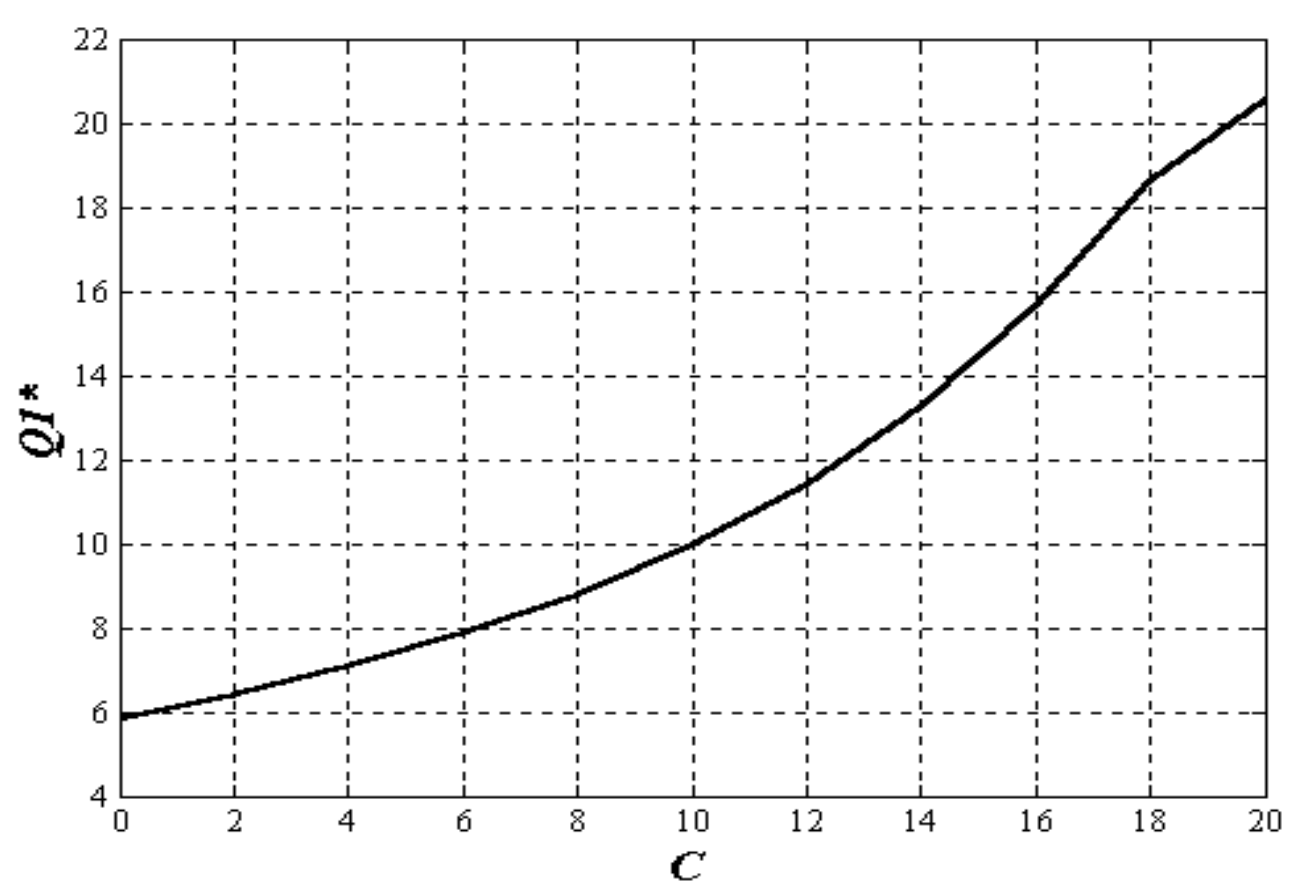

(a)

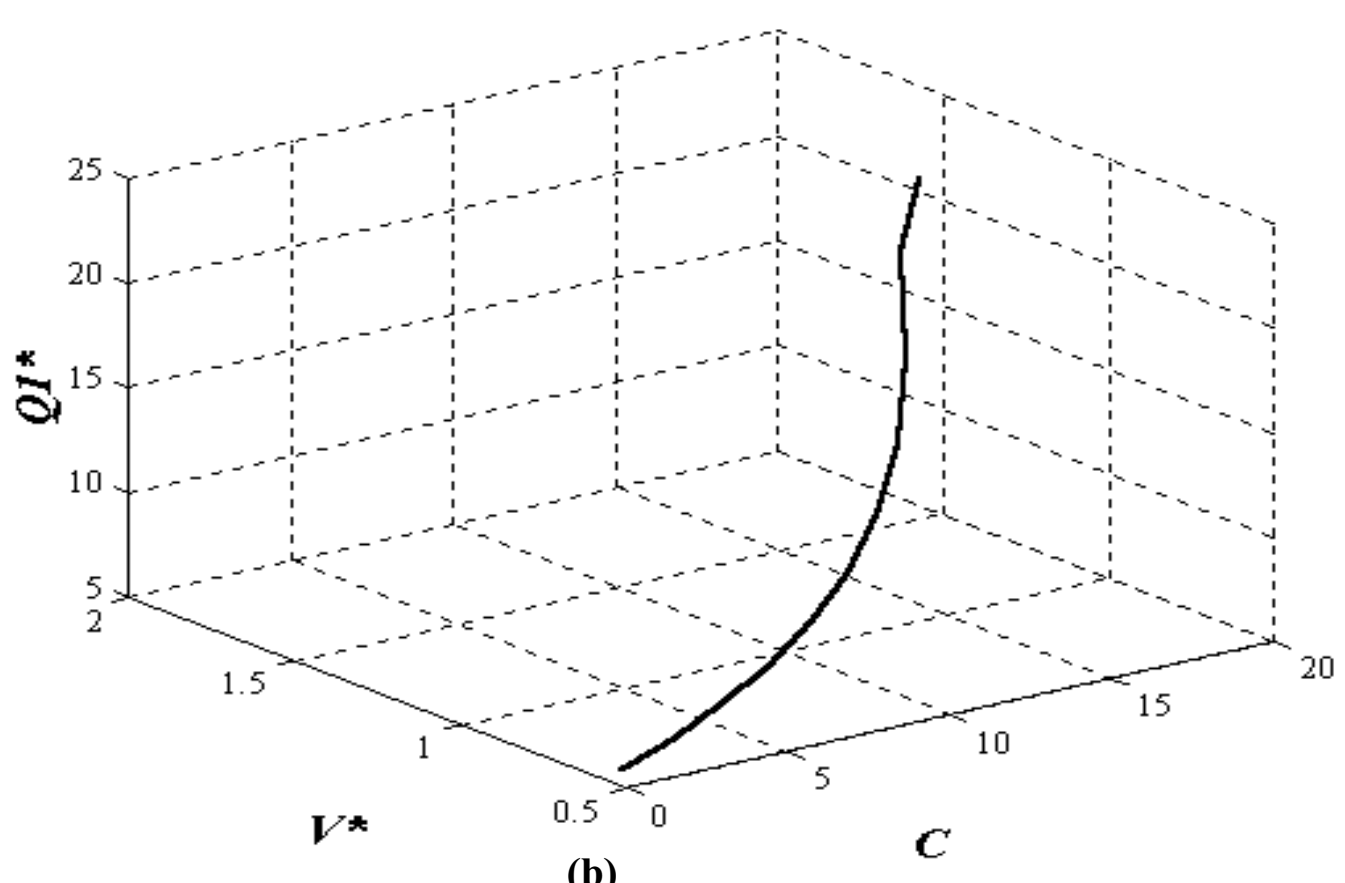

(b)

Fig.(6) Relationship between the installed capacity at the load bus and:

(a) The bifurcation value $\left(Q 1^{*}\right)$.

(b) The bifurcation value $\left(Q 1^{*}\right)$ and Voltage magnitude at bifurcation point. 


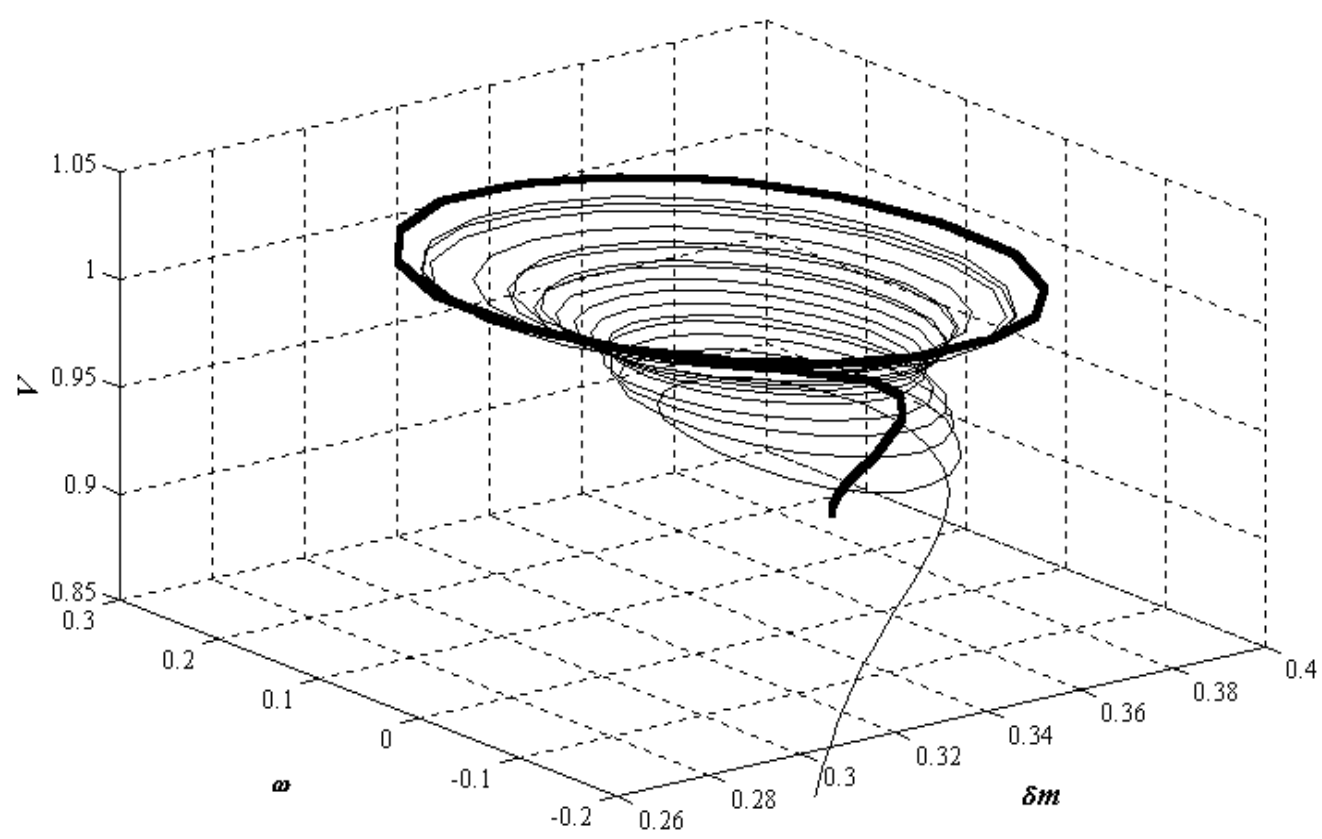

(a)

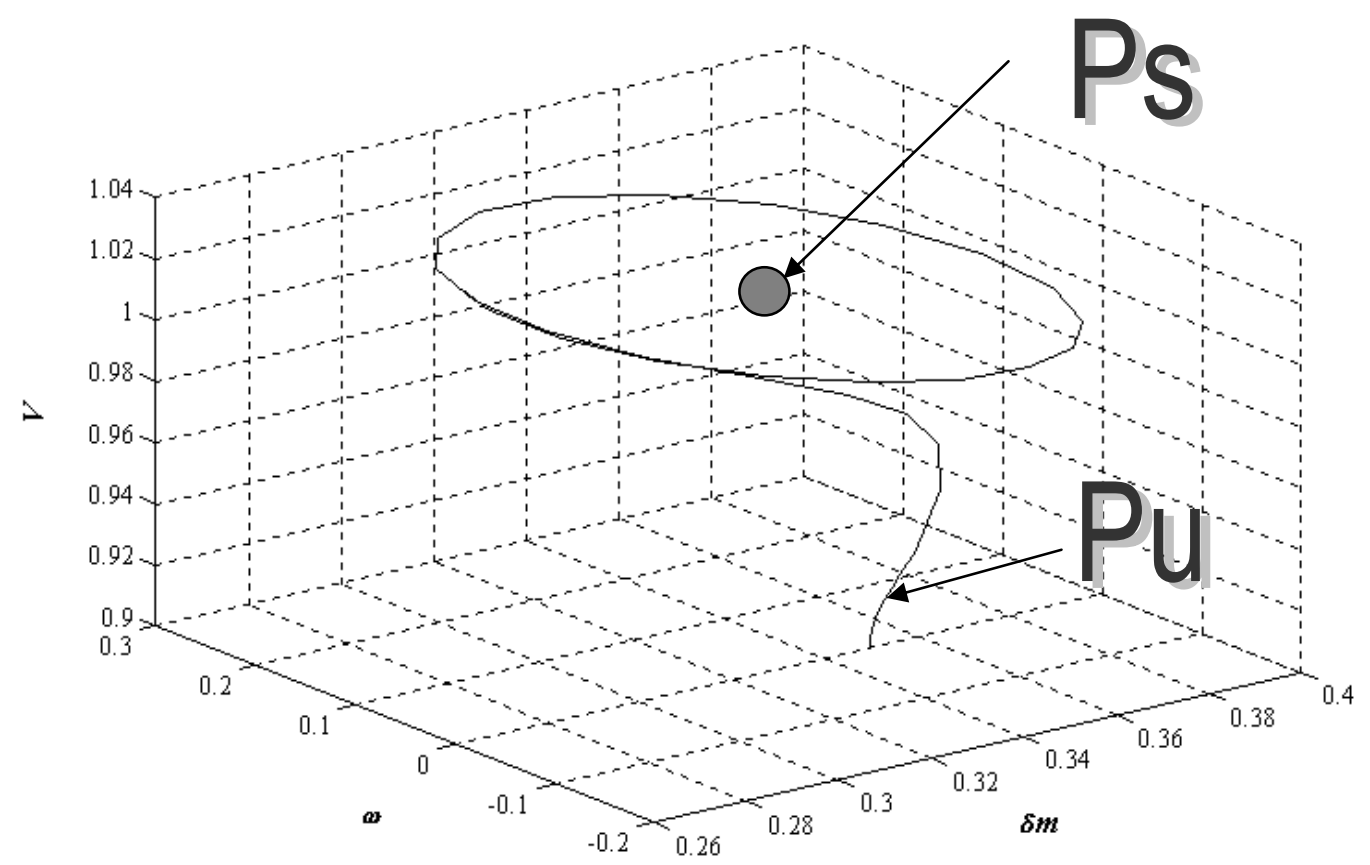

(b)

Fig.(7) Relationship between $\delta m \& \omega$, where:

(a) Unstable chaotic orbit for $Q 1=11.25+0.005 t$, simulation time $=32.919 \mathrm{sec}$.

(b)A stable periodic orbit for $Q 1=11.25+0.005 t$, simulation time $=2.65 \mathrm{sec}$.

Where Ps \& $\mathbf{P u}$ are points of stable and unstable respectively. 


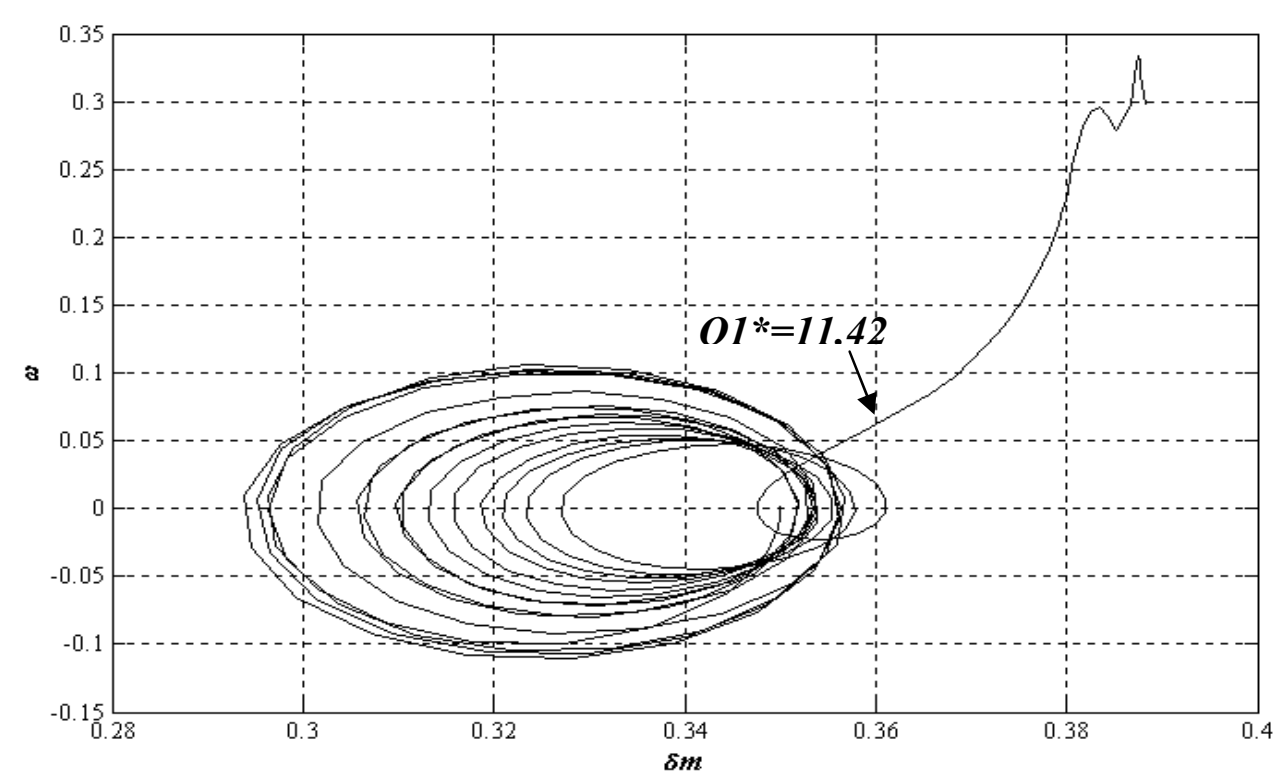

(a)

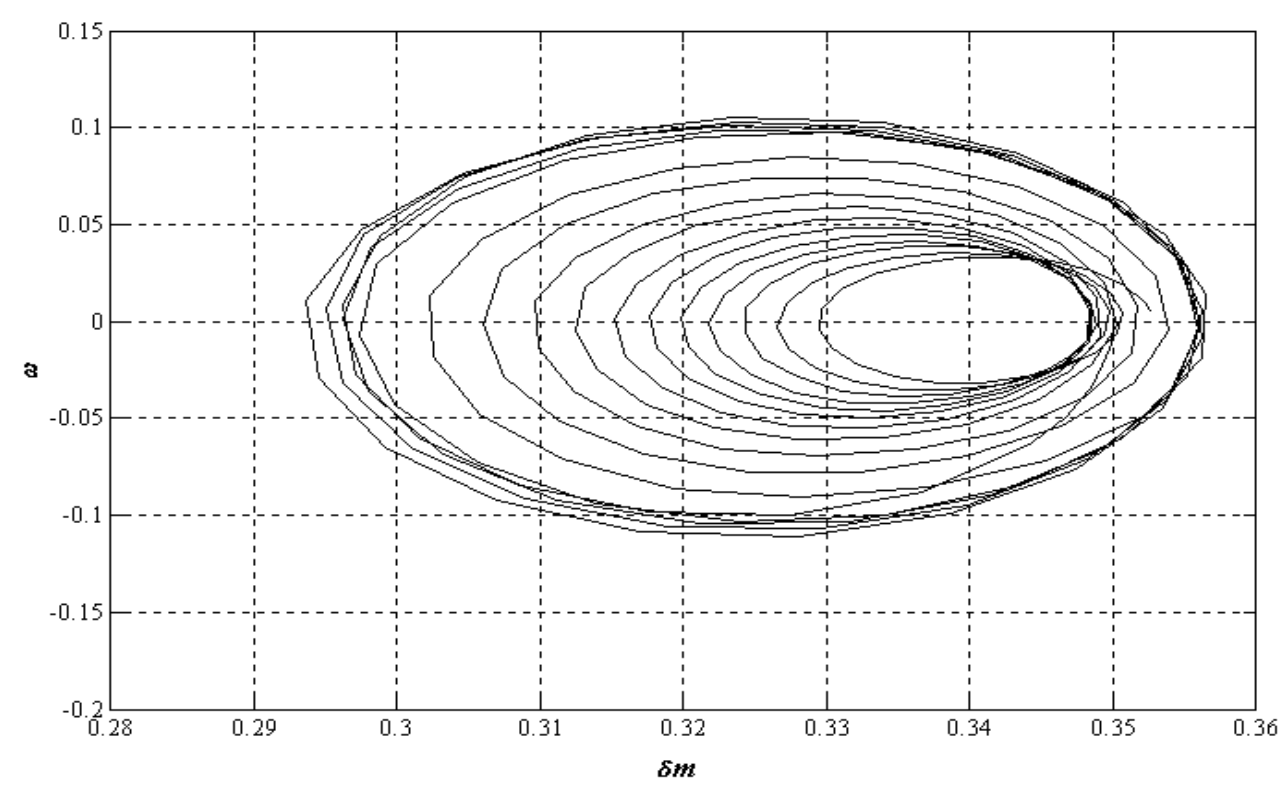

(b)

Fig.(8) Relationship between $\delta m \& \omega$ with simulation time $=30 \mathrm{sec}$, where:

(a) Unstable chaotic orbit for $Q 1=11.25+0.005658 t$.

(b)A stable periodic orbit for $Q 1>11.42$. 


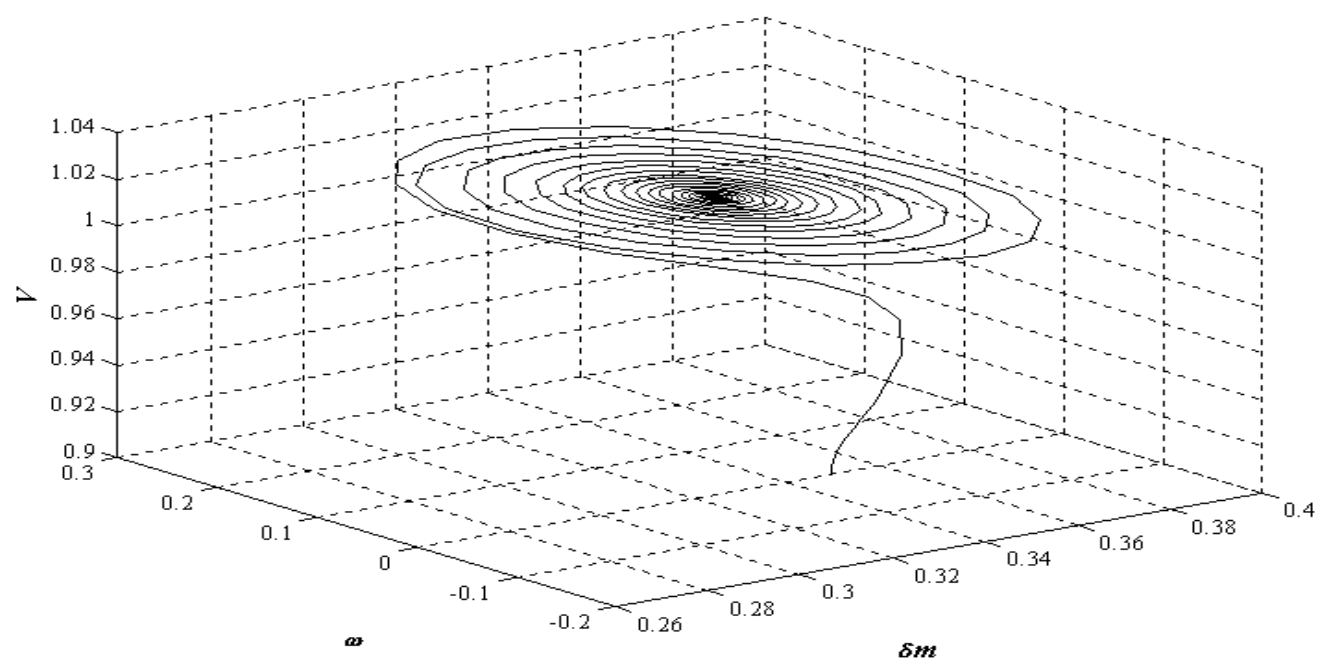

(a)

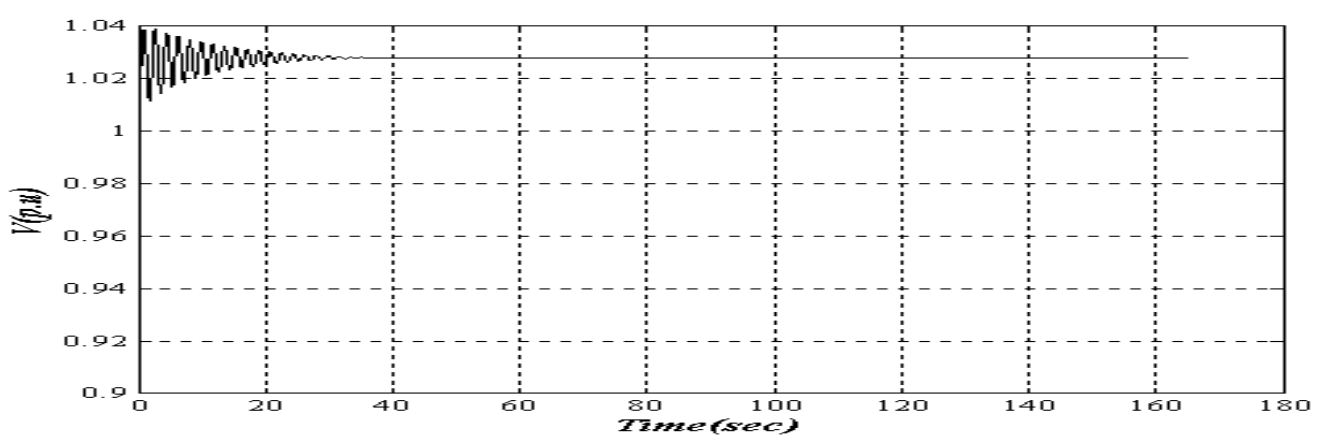

(b)

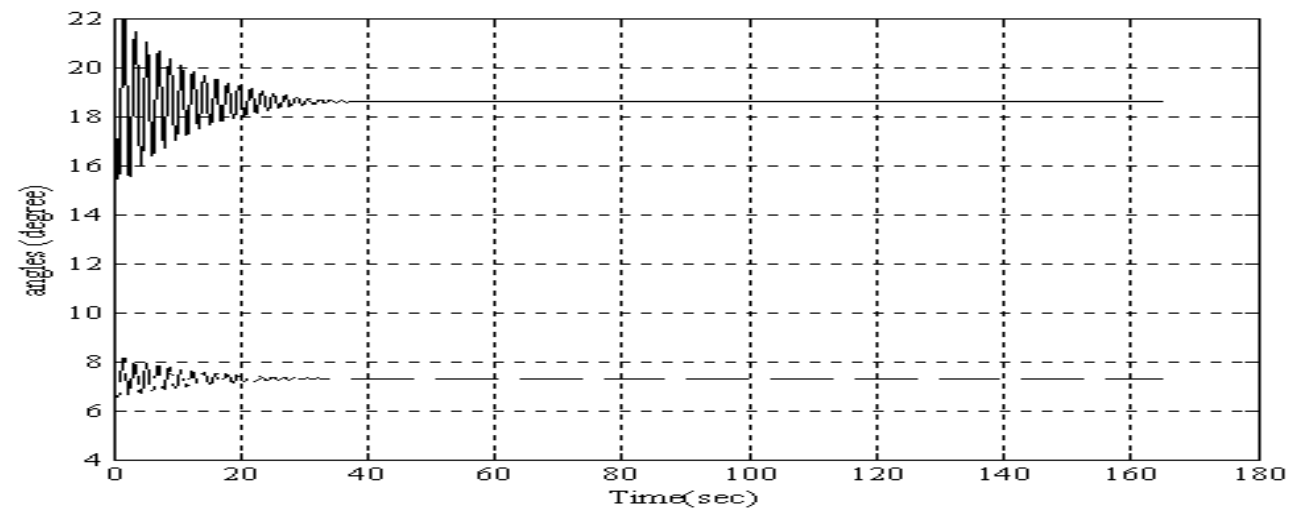

(c)

Fig.(9) The stable manifold periodic orbit with long simulation time $=180 \mathrm{sec}$, where:
(a) Relationship between $\delta \mathrm{m}, \omega \& \mathrm{~V}$.
(b) Relationship between V \& Time.
(c) Relationship between $\delta \mathrm{m}, \delta \&$ Time. 